\title{
Localization of cellulosic fines in paper via fluorescent labeling
}

\author{
Mathias A. Hobisch • Julie Bossu • Daniel Mandlez • Sylvia M. Bardet • \\ Stefan Spirk $\cdot$ Rene Eckhart $\mathbb{D} \cdot$ Wolfgang Bauer
}

Received: 27 March 2019/Accepted: 3 June 2019/Published online: 13 June 2019

(C) The Author(s) 2019

\begin{abstract}
Generated in the pulp and paper production process, cellulosic pulp fines are fibrous cellulosic materials capable of passing a 200 mesh screen. Processed pulp fines remain in the pulp and affect pulp and paper sheet properties. Their specific morphology promotes interactions with fibers and alters sheet properties. Nevertheless, the 3D distribution of fines in paper sheets has not been revealed so far. Localizing fines within a matrix of fibers is challenging since both elements have the same chemical composition and fines have small dimensions. It is required to increase the contrast of pulp fines, while avoiding any alteration of initial fines morphology. In this study, fines were first separated and labeled with Rhodamine $\mathrm{B}$ isothiocyanate, a fluorescent dye. Labeled fines
\end{abstract}

Electronic supplementary material The online version of this article (https://doi.org/10.1007/s10570-019-02556-0) contains supplementary material, which is available to authorized users.

M. A. Hobisch · J. Bossu - D. Mandlez .

S. Spirk $\cdot$ R. Eckhart $(\varangle) \cdot$ W. Bauer

Institute of Paper, Pulp and Fibre Technology, Graz

University of Technology, 8010 Graz, Austria

e-mail: rene.eckhart@tugraz.at

J. Bossu

UMR IATE, Universit de Montpellier, 34090 Montpellier,

France

S. M. Bardet

CNRS, XLIM, UMR 7252, University of Limoges,

87060 Limoges, France were blended with pulp at different concentrations and paper sheets were produced from each mixture. Prior to imaging, pulps and handsheets were characterized by standard pulp and paper tests and compared with references, showing no significant differences between sheets containing labeled and untreated fines. Thus, mechanical and physical tests indicated that no, or only minor alteration of fines properties by the labeling process occurred. We then applied two imaging techniques to detect the labeled pulp fines in the paper network, namely confocal laser scanning microscopy and multiphoton microscopy, visualizing the 3D distribution of fluorescent fines within the fiber network. The results obtained also allowed a differentiation between morphologically different fines showing fiber fragments more attached to single fibers whereas more fibrillar fines concentrate in fiber-fiber joints, thereby strengthening bonding. 


\section{Graphic abstract}

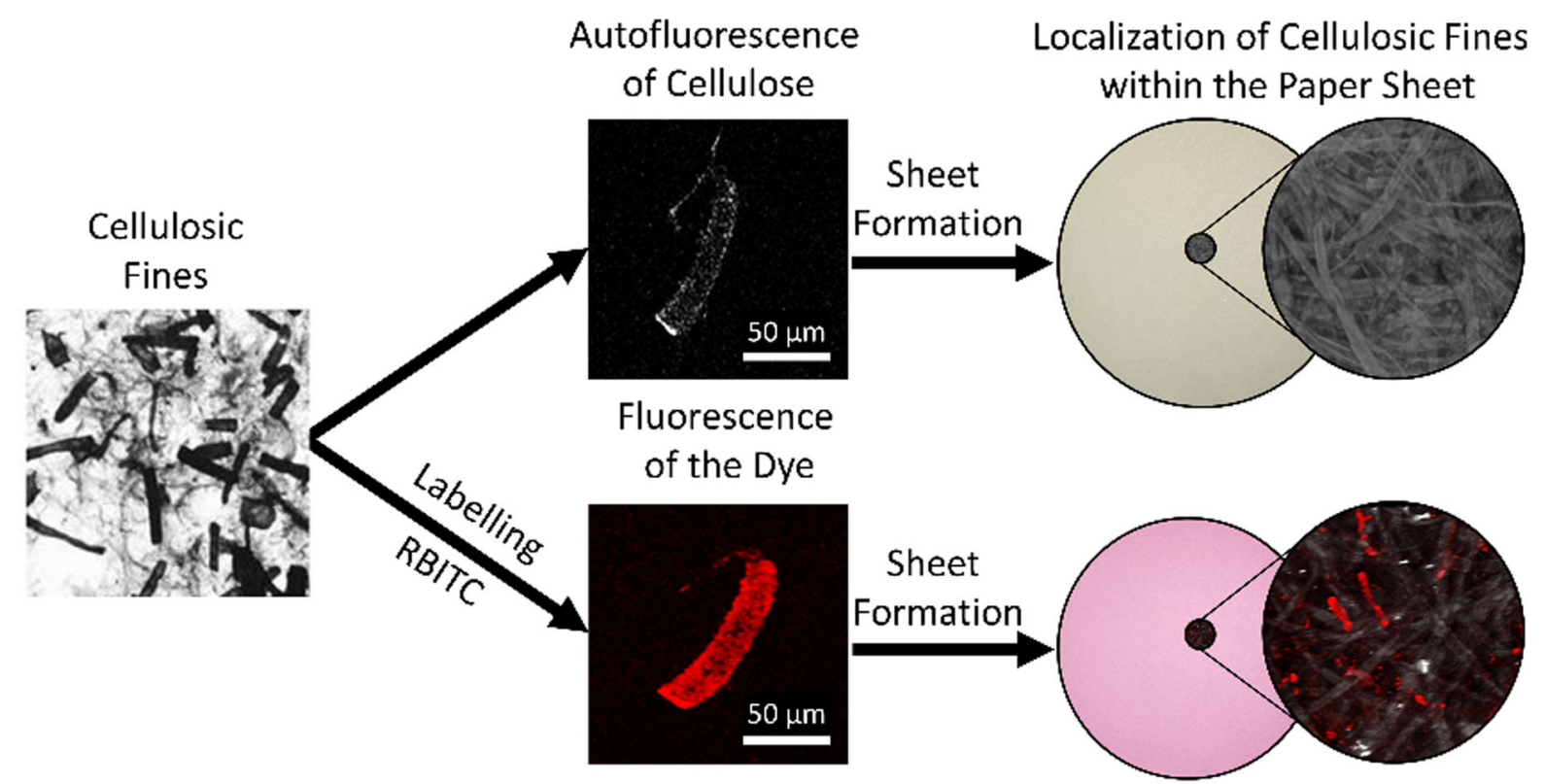

Keywords Cellulose - Paper - Cellulosic fines · Flourescent labeling · Confocal laser scanning microscopy $\cdot$ Multiphoton microscopy

\section{Introduction}

Natural fibers have a wide field of applications, for instance in paper and board, composites and insulating materials, textiles or as an energy source. Among these, paper production is the most significant industry forming sheets from individual fibers via a hierarchical network with defined properties demanded by the market.

Mechanical and chemical pulping processes isolate fibers from wood, preserving fibers as a suspension. The suspension passes several processing steps depending on the final application of the paper product. Pulping conditions and various chemical and mechanical steps lead to the formation of cellulosic microparticles, the so called pulp fines. By definition, pulp fines are able to pass a 200 mesh screen which is equivalent to holes with a diameter of $76 \mu \mathrm{m}$ (TAPPI 1994). In literature, pulp fines are further distinguished into primary and secondary fines. Primary fines are produced during pulping, generated under harsh chemical and mechanical conditions (Mark 1984). The average content of primary fines in chemical pulp is around 3 to 4 percent and they come as flake like structure, which originates from mostly ray cells and the middle lamellae (Westermark and Capretti 1988). Primary fines feature lower bonding ability compared to the more fibrillar secondary fines and affect the optical properties of the resulting papers. The following refining process generates up to $40 \%$ highly fibrillar secondary fines (Odabas et al. 2016). The secondary fines induce strong swelling and have a significant positive effect on mechanical strength by increasing the density of the final product while reducing air permeability. On the other hand the dewatering in the production process is more tedious. Although, primary and secondary fines exhibit a dominating morphology, both contain fiber segments or vice versa fibrillar parts to a lower extent (Mayr et al. 2017a). Summing up, fines in paper affect several paper properties such as shrinkage potential, sheet density and air permeability as well as mechanical properties (Odabas et al. 2016).

Most of the publications in this field report the advantages of smaller cellulosic components in the improvement of the final product technological performances. Herein, the advantages of fines are correlated with specific paper mechanical properties . 
Nonetheless, why and how said differences in morphology affect the technological properties, remain to some extent a matter of speculation. Indeed, the distribution of fines within the fiber network and their contribution to the network was recently elaborated, without distinguishing between fiber segments and more fibrillar parts of paper fines (Hobisch et al. 2019). This observation not only applies to the above discussed fines categories but also to micro (MFC)and nanofibrillated cellulose (NFC), which are already commercially available and used as strengthening agent (Bossu et al. 2019) to improve surface properties of paper, as described in recent reviews of possible applications of micro- (Osong et al. 2015) and nanofibrillated cellulose (Boufi et al. 2016).

In plant research, confocal laser scanning microscopy (CLSM) and multiphoton microscopy (MPM) are emerging non-invasive techniques to visualize cell wall components. For distinction, both techniques require the use of fluorescent dyes such as Rhodamine B isothiocycanate (RBITC, emission at $595 \mathrm{~nm}$ ) that are covalently bound to the lignocellulosic material of interest (Ding et al. 2018). In general, lignocellulosics feature autofluorescence in plant-derived materials that can be detected with linear (Lundquist et al. 1978) or nonlinear excited fluorescence techniques.

So far, CLSM has gained popularity in the scientific community to study several paper-related properties, such as the orientation of fibers in paper sheets (Enomae et al. 2008). Moss et al. studied the distribution of stained thermomechanical pulp (TMP) fines in a chemical pulp network (Moss et al. 1993). Contrary to their expectations, this study revealed that TMP fines were evenly distributed within the sheet while the authors could not detect a higher amount of fines close to fiber-fiber bonds.

MPM gives the possibility to combine different modes of contrast, mainly two-photon excited fluorescence from fluorophores and second harmonic generation from non-centrosymmetric structures (Brown et al. 2003). The second harmonic generation is a nonlinear optical process. Two photons with the same frequency interact with a nonlinear material and generate a new photon featuring twice the energy of the two initial photons (equivalently, twice the frequency and half the wavelength). Combining the two modes thus enables the study of detailed components of vegetal fibers by revealing crystalline cellulose (second harmonic generation channel) and lignin (autofluorescence, two-photon excited fluorescence channel) in the cell walls, as reported previously for celery petiole (Cox et al. 2005) and wood (Latour et al. 2012).

The objective of this work was to apply this labeling technique and to detect fines within the fiber network. CLSM, as well as MPM showed their operational capability providing further insights into lignocellulosic structures. Additionally, their world wide availability and fast processing makes them an interesting approach for the localization of labeled cellulose microparticles.

The workflow of the study is as follows: First, the fines were separated from the pulp using a pressure screen (I). After separation, pulp fines were labeled with Rhodamine B isothiocyanate (II) with the purpose of increasing the contrast in the cellulosic network. Paper fibers and fines were blended (III), and sheets containing the labeled fines were formed (IV). Physical and mechanical properties of the pulp and sheets were investigated (V) prior to imaging (VI). Herein, CLSM and MPM revealed similar structures highlighting fiber segments as well as highly fibrillar fines material within the sheet.

\section{Experimental}

\section{Materials}

Epichlorohydrin (>99\%) and ammonium chloride/ ammonium hydroxide buffer solution $\left(\mathrm{NH}_{4} \mathrm{Cl}, 1 \mathrm{wt} \%\right.$; $\mathrm{NH}_{4} \mathrm{OH}, 4 \mathrm{wt} \%$ (pH 10 to 11) were supplied by Sigma-Aldrich (Vienna, Austria), $\mathrm{NaOH}$ (99\%) was obtained from VWR chemicals (Radnor, USA) and Rhodamine B isothiocyanate (RBITC; mixed isomers) was purchased from Cayman Chemical (Ann Arbor, USA). The paper pulp originates from an industrial process (ORION, Heinzel pulp—Zellstoff Pöls AG, Pöls, Austria). All chemicals were used without further purification.

\section{Separation of pulp fines}

The Schopper-Riegler method evaluates the dewatering of the pulp suspension in $\mathrm{SR}^{\circ}$ and is a parameter indirectly describing the morphological development of the pulp during refining (Gockel \& Co, Type 401, according to ISO 5267-1). Pulp fines were separated 
from a refined bleached softwood Kraft pulp (BSK; $38^{\circ} \mathrm{SR}$ ), using a pressure screen built for this purpose with a $100 \mu \mathrm{m}$ hole screen (Fischer et al. 2017). These fines represent a mixture of primary and secondary fines. The separated fines were concentrated using flotation to produce a suspension with $\sim 0.8 \%$ dry content. The same bleached softwood Kraft pulp $\left(16^{\circ} \mathrm{SR}, 2000\right.$ revolutions in the PFI-mill, tensile properties comparable to industrial application of said pulp) was used as pulp matrix in the trials. The fines content of this pulp, evaluated by Dynamic Drainage Jar, was $4.5 \%$. These fines were almost exclusively primary fines as no secondary fines were produced yet in PFI treatment at the given $\mathrm{SR}^{\circ}$ value.

\section{Preparation of fluorescent fines (FF)}

The functionalization of pulp fines followed the routine of Ding et al. 2018 and was visualized in Scheme 1. First, the activation of the fines $(\sim 0.8 \%)$ was conducted by introducing an epoxy group under controlled alkaline conditions $(\sim \mathrm{pH} 12)$ by adding epichlorohydrin $\left(5 \mathrm{ml} \mathrm{g}^{-1}\right.$ fines) under steady stirring for $2 \mathrm{~h}$ at $60{ }^{\circ} \mathrm{C}$. The suspension was centrifuged and washed with deionized water. Second, an amino group was introduced onto the cellulose through the addition of an ammonium chloride ammonium hydroxide buffer ( $\mathrm{pH} \quad 10-11 ; 5 \mathrm{ml} \mathrm{g}^{-1}$ fines) under steady stirring at $60{ }^{\circ} \mathrm{C}$. The suspension was extensively washed to remove all the excessive ammonium chloride. Third, the cellulose was functionalized by adding RBITC ( $0.01 \mathrm{~g} \mathrm{~g}^{-1}$ fines), stirring the solution for $24 \mathrm{~h}$ at room temperature. Afterwards, the fines were rinsed with distilled water to remove the surplus of the dye not attached to the surface, controlling the process via UV/VIS-measurement of the filtrate. The zeta potential of the pulp fines was measured with the Stabino ${ }^{\circledR}$ device (Particle Metrix, Stabino, Meerbusch, Germany) in distilled water comparing untreated and fluorescent fines at $\mathrm{pH} 7$.

\section{Sheet formation and testing}

The pulp was mixed with $1 \%$ and $2 \%$ untreated (BSK1, BSK2) and labeled fines (FFBSK1 and FFBSK2). The water retention value (ISO 23714:2014) of these blends was determined. The swollen pulp was centrifuged at $3000 \mathrm{~g}$, weighed, dried at $105{ }^{\circ} \mathrm{C}$ and reweighed. Herein, the water retention value represents the ratio of water retained in the pulp after centrifugation in $\mathrm{g} / \mathrm{g}$. In order to get a defined pulp fines ratio in the hand sheets, we used a Rapid-Köthen sheet former (FRANK-PTI, ISO 5269-2:2004) combined with white water recirculation according to a published procedure (Giner Tovar et al. 2015). The first five sheets were discarded until a stable fines content (representing 100\% retention) in the sheets was achieved. Seven sheets were formed $\left(60 \mathrm{~g} \mathrm{~m}^{-2}\right)$ and wet pressed (150 bar, $\left.90 \mathrm{~s}\right)$ between two blotting papers prior to sheet drying. The sheets were stored $24 \mathrm{~h}$ in a climate room $\left(23{ }^{\circ} \mathrm{C} ; 50 \% \mathrm{RH}\right)$ before testing. The tested parameters were air permeability according to Bendtsen (ISO 5636-3:2013), density (ISO 534:2011) and tensile index (ISO 1924-2:2008, FRANK-PTI tensile tester). In addition, the isoelectric point was evaluated in $1 \mathrm{mM} \mathrm{KCl}$ solution, by titrating the suspension with a $0.05 \mathrm{M} \mathrm{HCl}$ using the SurPASS 3 (Anton Paar, Austria).

\section{Confocal laser scanning microscopy}

Sheets and fluorescent fines (FF) were visualized by confocal laser scanning microscopy (FISH/CLSM) using a Leica TCS SPE confocal laser scanning microscope (Leica Microsystems, Mannheim, Germany) with oil immersion objective lenses Leica ACS APO $10.0 \times \mathrm{CS}$, exciting cellulose at $405 \mathrm{~nm}$ and RBITC at $532 \mathrm{~nm}$. The emission spectra between 420 and $500 \mathrm{~nm}$ visualizes the auto-fluorescence of cellulose superimposed by the emission spectra of FF which was determined between 550 and $600 \mathrm{~nm}$.

\section{Multiphoton microscopy}

Two-photon excitation microscopy was also used for fluorescence analysis. Paper sheets were positioned on a stage of a customized Olympus multiphoton microscope BX61WI/FV1200MPE with a 25X immersion objective (1.05NA, $2.0 \mathrm{~mm}$ working distance) coupled with a tunable femtosecond Ti:Sapphire pulsed laser (Chameleon Ultra II, Coherent) for the excitation (Bardet et al. 2016). Image stacks were acquired under $810 \mathrm{~nm}$ excitation for second harmonic generation (cellulose) and fluorescence (RBITC for fines, autofluorescence for lignin) wavelength with FluoView FV1200 software (v4.1.1.5, Olympus). Each acquisition in photon-counting mode produces a 3D stack of $640 \times 640 \times 53$ pi, with a sampling step of $2 \mu \mathrm{m}$ and 
a dwell time of $20 \mu \mathrm{si}^{-1}$ (input laser $20 \mathrm{~mW}$ ). The different components of the emitted light from the sample were separated using a dichroic mirror $(450 \mathrm{~nm})$ and detected by a pair of photomultiplier tubes preceded by fluorophore specific emission filters (607/36 for fluorescence in red, 405/10 for second harmonic generation in green). The obtained images were analyzed with Imaris software (Bitplane AG) or Fiji/ImageJ (NIH).

\section{Results and discussion}

Efficiency of fluorescent labeling on fines

Fines were labeled with RBITC after separation from the paper pulp (Fig. 1).

Optical microscopy and CLSM of functionalized labeled fines were performed to distinguish between autofluorescence of lignocellulosic fibers (Fig. 2a) and the fluorescence signal emitting at $595 \mathrm{~nm}$ from RBITC (Fig. 2b) induced by covalent attachment of RBITC. Further, the images revealed that the fluorescent labelling is homogenous throughout the whole surface of the fines. The zeta potential of fines and labeled fines further proved the successful labelling of the fines revaling a zeta potential close to $0 \mathrm{mV}$ while the unmodified fines featured a zeta potential of $-30 \mathrm{mV}$.

Influence of fluorescent labeling on sheet properties

Relevant physical and mechanical properties were compared between paper sheets containing no additional (blank), unlabeled (BSK1 and BSK2) and fluorescent fines (FFBSK1 and FFBSK2). The blank, BSK1 and BSK2 exhibited the same brightness, while FFBSK2 appeared visually slightly more intense in color than FFBSK1. This is caused by the higher concentration of labeled fines in those sheets. Nevertheless, labeled fines neither affected the zeta potential, nor the isoelectric point of the handsheets (Table S1). The water retention values of the pulps (Fig. 3a) increased from BSK1 to BSK2, which relates to the higher concentration of fines in the latter which retain more water (Mayr et al. 2017b). FFBSK1 and FFBSK2 showed slightly higher ability to retain water compared to BSK1 and BSK2, regardless of the concentration. While FFBSK2 was in the confidence interval of BSK2, FFBSK1 was beyond the one of BSK1. The increased water retention value (WRV) indicates more pronounced swelling that corresponds to the higher density of the paper sheets (Fig. 3b).

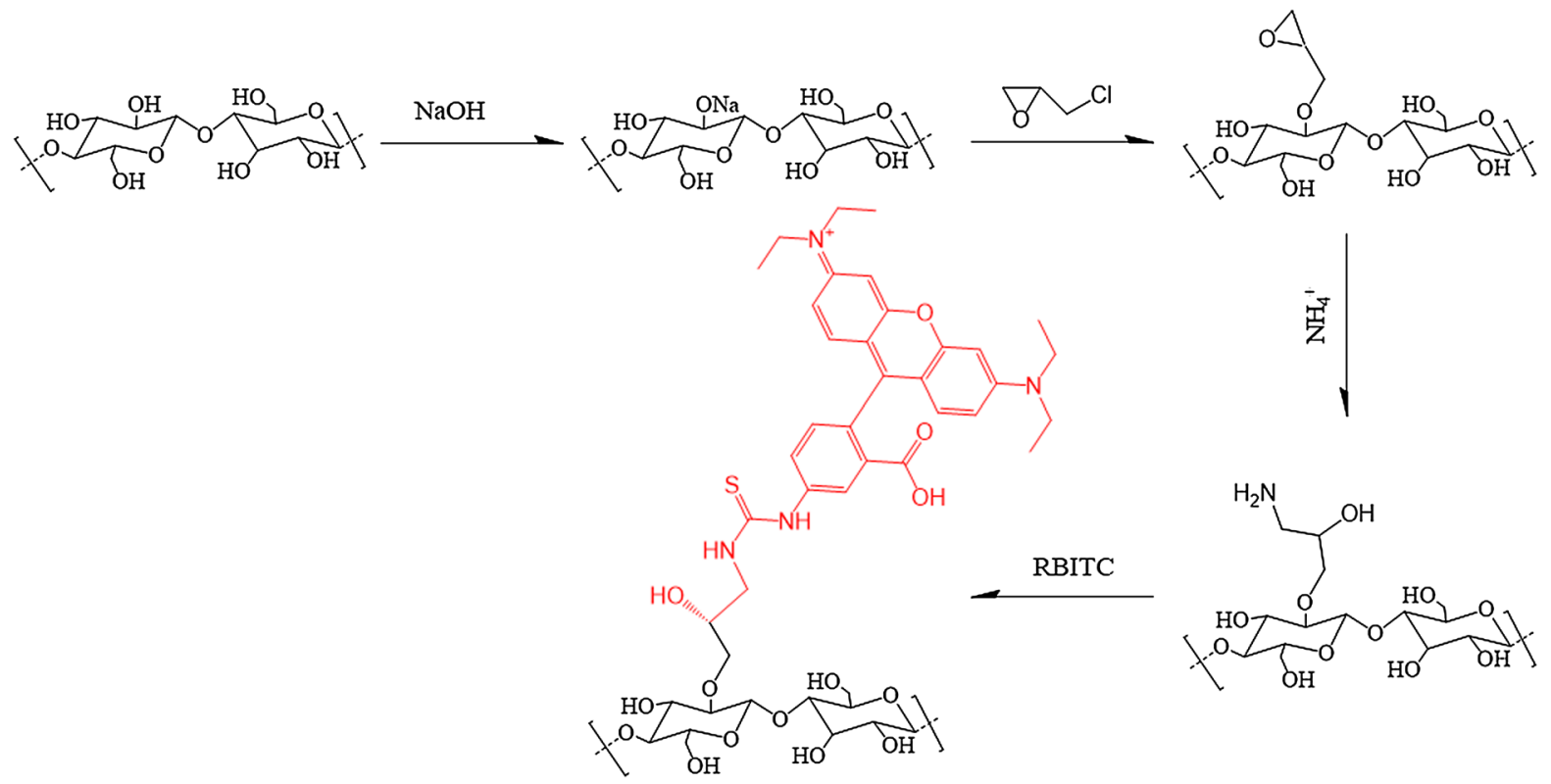

Fig. 1 Fluorescent labeling procedure for paper fines 

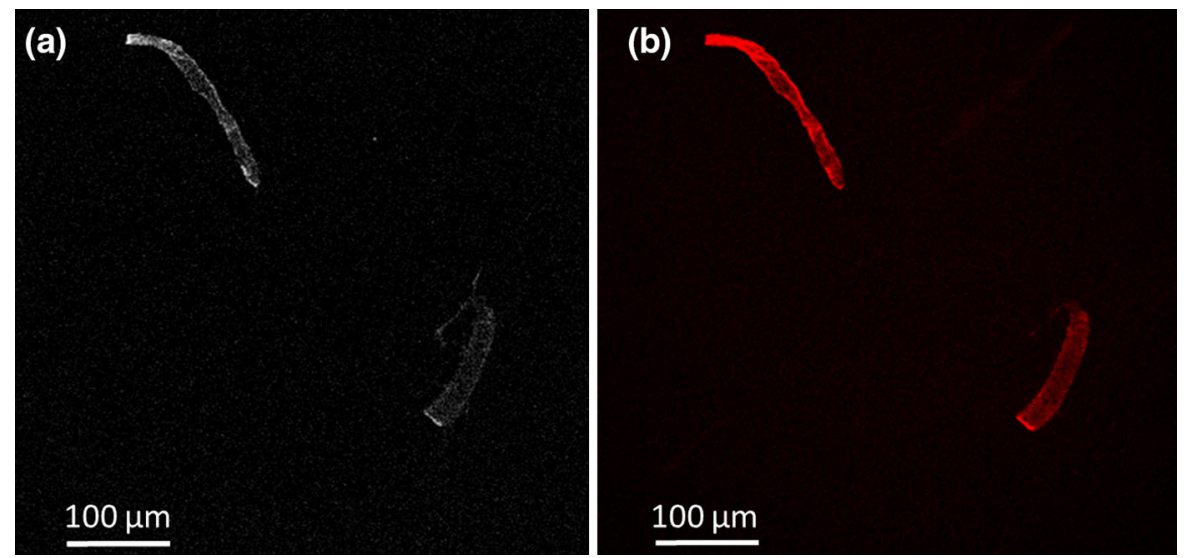

Fig. 2 CLSM images visualizing a autofluorescence and $\mathbf{b}$ the fluorescent dye RBITC of labeled fines
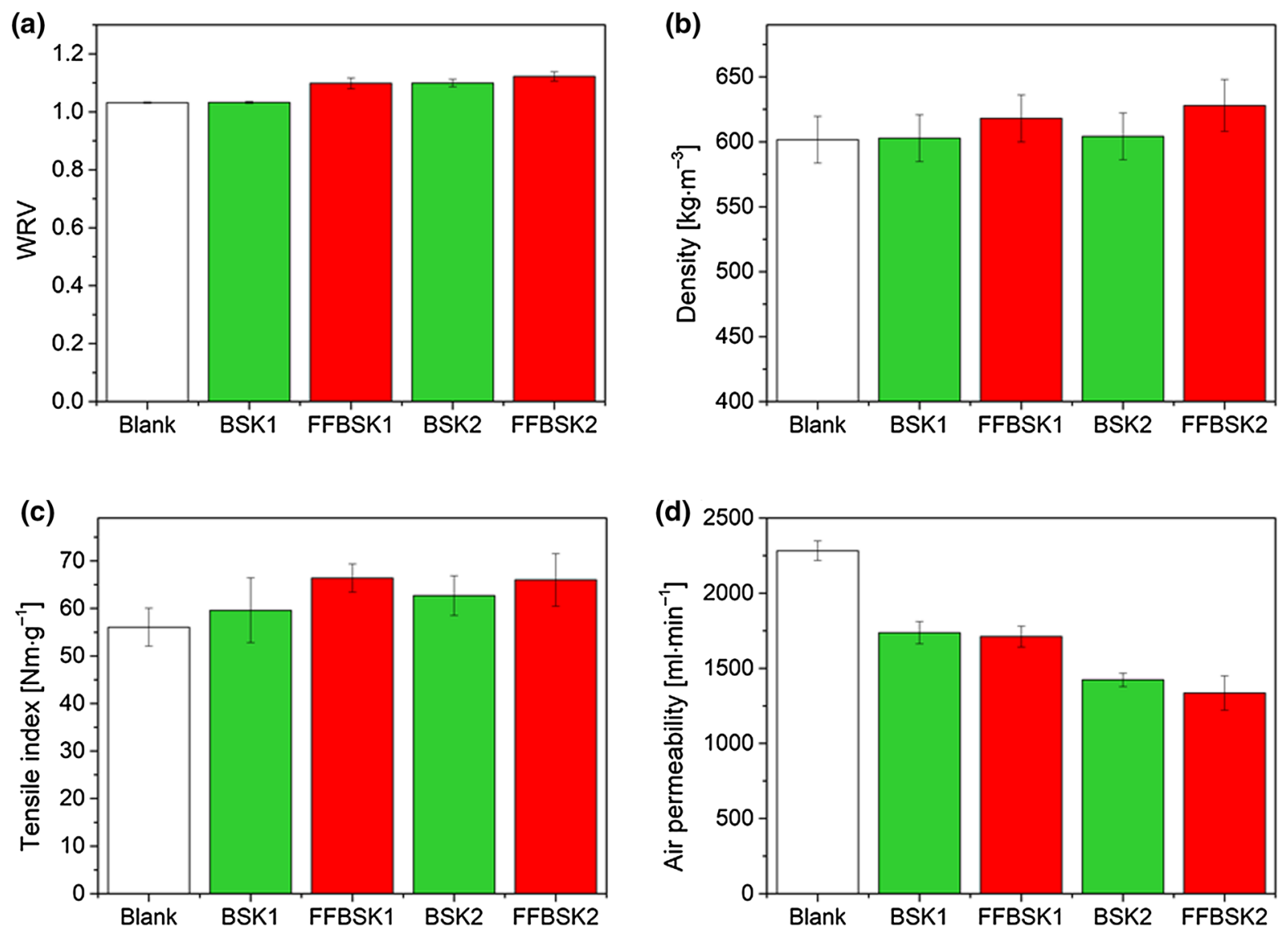

Fig. 3 Pulp/handsheets containing $1 \%$ and $2 \%$ of untreated (green) and fluorescent labeled fines (red) were examined concerning a sheet density, $\mathbf{b}$ water retention value, $\mathbf{c}$ tensile index and $\mathbf{d}$ air permeability

Consequently, the higher density is reflected in the mechanical properties (Fig. 3c) and air permeability (Fig. 3d) as well. Nevertheless, the differences between unlabeled and labeled fines in handsheet density, mechanical properties and air permeability were not significant, when taking the corresponding confidence intervals (95\% level of confidence) into account. 
Furthermore, sheet formation was investigated by beta radiography. The results revealed no significant change in mass distribution, which indicates that flocculation does not seem to be impaired (Fig. S1). Therefore, the labeled fines should represent the properties of unlabeled ones well and may interact with the paper network in a similar way. Studying these interactions shall therefore give new insights into the role of fines within the network and their technological impact.

Localization of fines within the paper matrix

All samples were analyzed by confocal laser scanning microscopy (CLSM) and multiphoton microscopy (MPM). Both techniques should allow for the localization of fibers and fines within the $\mathrm{x}-, \mathrm{y}-$ and $\mathrm{z}$-direction of the handsheets.

With CLSM, both, labeled fines and the pulp fibers, were excited at $405 \mathrm{~nm}$ and the emission was recorded between 420 and $500 \mathrm{~nm}$, corresponding to autofluorescence. The detected autofluorescence (grey areas in Fig. 4) clearly reflected the fiber network of the studied paper samples. In these measurements, any differences for paper sheets containing $1 \%$ and $2 \%$ fines (i.e. BSK1 and BSK2, Fig. 4a) were not observed. Excitation at $532 \mathrm{~nm}$ in turn, just led to noisy signals for sheets made from BSK1 and BSK2 (Fig. S2). In contrast, FFBSK1 and FFBSK2 labeled fines gave a clear response after excitation at $532 \mathrm{~nm}$ and allowed for their visualization inside the paper. This further proves that the fluorescent labeled fines can be easily differentiated from the non-labeled cellulosic matrix. A considerable increase of labeled segments is evident in Fig. 4b, c, which is consistent with the increased amount of labeled fines. Obviously, the labeled fines are well distributed within the fiber network. Based on such measurements also 3D investigations and analysis of cross sections are possible with resolution limited by the light refraction in z-direction. Such investigations could enable the characterisation of the segments within the fiber joints. At this point, CLSM reaches its resolution limits for paper sheets.

The application of MPM extends the investigations of fluorophores as labeling technique in paper sheets reducing the blurring by exciting the fluorophore with two photons at the same time, while suppressing the out-of-focus light. The reduction of out-of-focus light enables deeper insight into the cellulosic structure (second harmonic generation) while increasing simultaneously the resolution. The second harmonic generation from cellulose was measured between 400 and $410 \mathrm{~nm}$ (green) superimposed by the fluorescence signal of the labeled fines between 589 and $625 \mathrm{~nm}$ (red). BSK1 and BSK2 that only contained nonlabeled fines (Fig. 5a) clearly revealed a fiber network, while the fluorescence signal results in noise, similar to the one in Fig. 4a. Superimposing the second harmonic generation with the fluorescence signal of FFBSK1 and FFBSK2 showed particles with a specific structure. Larger particles (white arrows) and smaller particles (yellow arrows) are visible (Figs. 5, S3).
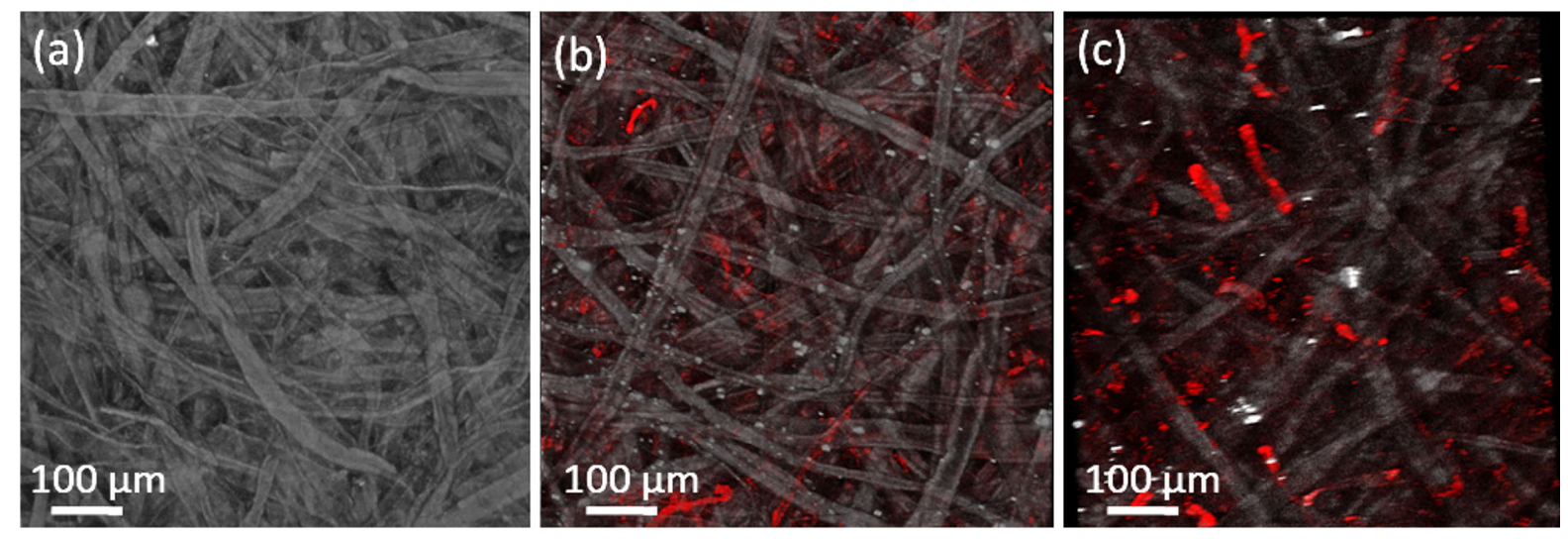

Fig. 4 CLSM images of different paper sheets. a BSK1, b FFBSK1 and c FFBSK2. Non-labeled cellulose fibers appear in grey and fluorescent labeled fines in red 

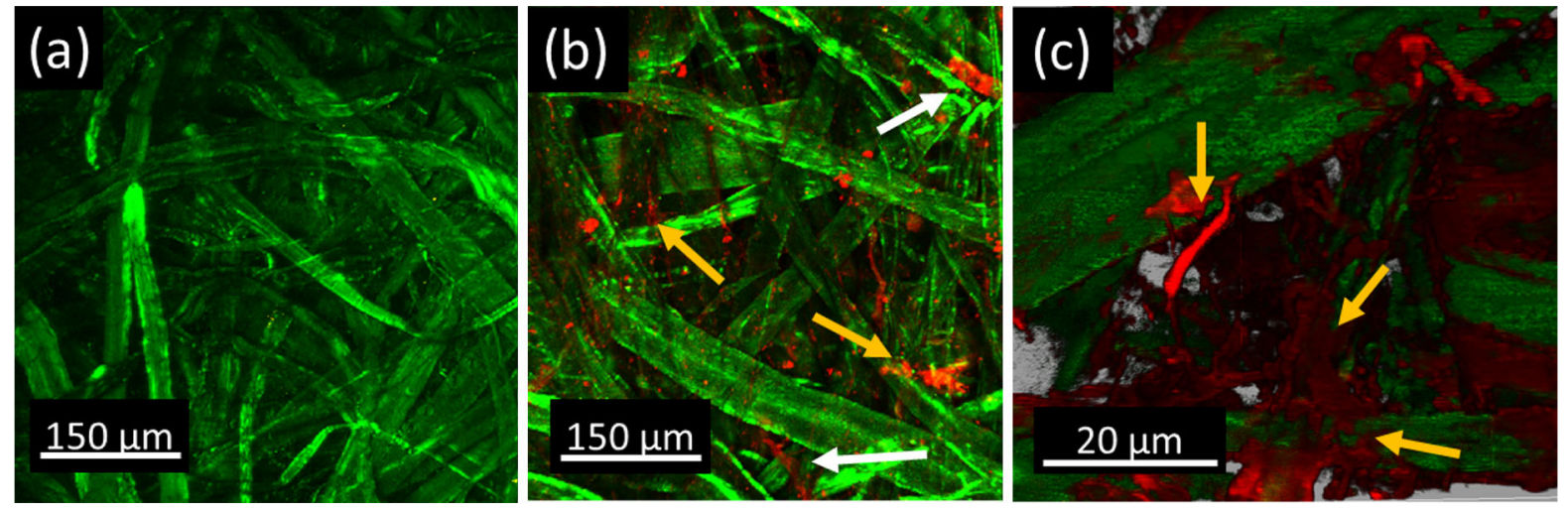

Fig. 5 Simultaneous fluorescence/second harmonic generation acquisitions have been performed by 2-photon microscopy images of FFBSK sheets containing $\mathbf{a} 0 \%$ and $\mathbf{b} 2 \%$ labeled fines at magnification of $\times 150$, and containing $1 \%$ fines at $600 \mathrm{X}$. Cellulose fibers (second harmonic generation) and fluorescent fines (RBITC) appear respectively in green and red within the

Herein, the larger fiber segments (primary fines) seem to participate in the network just like pulp fibers do, in general, due to their size. Smaller particles (secondary fines) on the other hand seem to be more concentrated in specific spots. Due to their size, they are not limited to the same extent from moving within the structure. Therefore, they are allowed to migrate within the network, most probably following the retreating water during dewatering into the smallest interstices between fibers, which later on form fiberfiber joints. These small particles are attached to a cellulose fiber and the fiber-fiber joint, as evident in the bottom of the image that is wrapped by fluorescent fines, tagged with orange arrows in Fig. 5c. This accumulation in the fiber-fiber joints supports the idea of Retulainen (Retulainen et al. 1993), who proposed less free loops (unbonded fiber segments) at higher fines concentration. Higher fines concentrations also promote accumulation, especially fibrillar parts. Hence, secondary fines have a much bigger impact on paper properties than primary fines (Bäckström et al. 2008; Chen et al. 2009).

\section{Conclusion}

The interactions of pulp fines in paper sheets were investigated by developing a labeling procedure for these small cellulosic constituents in order to visualize them in a coherent matrix investigating their paper sheet. The autofluorescence from the lignin component overlaps with the Rhodamine B fluorescence. Herein, white arrows assign larger fiber segments (presumably primary fines) and orange ones fine fibrillar particles (presumably secondary fines)

distribution, form and impact within the sheets. Pulp fines were labeled with Rhodamine B isothiocyanate to increase the contrast within the sheet. The use of two independent microscopic techniques-confocal laser scanning microscopy and two-photon microscopy-allows for the investigation of interactions between fibers and labeled fines within the paper network, thereby gaining further knowledge on their impact on technological paper properties. Furthermore, labeling allows differentiation between primary and secondary fines based on visualization of larger fiber segments (presumably primary fines) more attached to single fibers whereas more fibrillar parts (presumably secondary fines) concentrate in fiberfiber joints strengthening the bonding between two adjacent fibers. The imaging systems enable the introduction of new theories concerning the impact of paper fines on paper properties and can easily be extended to other cellulosic materials applied in the paper and packaging industry, which makes it a promising routine for future investigations.

\section{Supporting information}

Additional characterization (beta radiography, additional CLSM images, additional MPM images), Table S1, Fig. S1-S4. 
Acknowledgments Open access funding provided by Graz University of Technology. The authors acknowledge the industrial partners Sappi Gratkorn, Zellstoff Pöls and Mondi Frantschach, the Austrian Research Promotion Agency (FFG) (Grant No. 861476), COMET, BMVIT, BMWFJ, the Province of Styria and Carinthia for their financial support of the K-project Flippr ${ }^{2}$-Process Integration. This work was supported by grants from the LabEX SigmaLim (ANR-10-LABX-007401). We also thank Inge Mühlbacher, who proved insight and expertise that greatly assisted this research.

\section{Compliance with ethical standards}

Conflict of interest The authors declare that they have no conflict of interest.

Open Access This article is distributed under the terms of the Creative Commons Attribution 4.0 International License (http:// creativecommons.org/licenses/by/4.0/), which permits unrestricted use, distribution, and reproduction in any medium, provided you give appropriate credit to the original author(s) and the source, provide a link to the Creative Commons license, and indicate if changes were made.

\section{References}

Bäckström M, Kolar M-C, Htun M (2008) Characterisation of fines from unbleached kraft pulps and their impact on sheet properties. Holzforschung 62:546-552. https://doi.org/10. 1515/hf.2008.081

Bardet SM, Carr L, Soueid M, Arnaud-Cormos D, Leveque P, O'Connor RP (2016) Multiphoton imaging reveals that nanosecond pulsed electric fields collapse tumor and normal vascular perfusion in human glioblastoma xenografts. Sci Rep 6:34443. https://doi.org/10.1038/srep34443

Bossu J, Eckhart R, Czibula C, Winter A, Zankel A, GindlAltmutter W, Bauer W (2019) Fine cellulosic materials produced from chemical pulp: the combined effect of morphology and rate of addition on paper properties. Nanomaterials (Basel) 9:321-338. https://doi.org/10.3390/ nano9030321

Boufi S, Gonzalez I, Delgado-Aguilar M, Tarres Q, Pelach MA, Mutje P (2016) Nanofibrillated cellulose as an additive in papermaking process: a review. Carbohydr Polym 154:151-166. https://doi.org/10.1016/j.carbpol.2016.07. 117

Brown JRM, Millard AC, Campagnola PJ (2003) Macromolecular structure of cellulose studied by second-harmonic generation imaging microscopy. Opt Lett 28:2207-2209. https://doi.org/10.1364/ol.28.002207

Chen H, Park A, Heitmann JA, Hubbe MA (2009) Importance of cellulosic fines relative to the dewatering rates of fiber suspensions. Ind Eng Chem Res 48:9106-9112. https://doi. org/10.1021/ie9006613

Cox G, Moreno N, Feijo J (2005) Second-harmonic imaging of plant polysaccharides. J Biomed Opt 10:024013. https:// doi.org/10.1117/1.1896005
Ding Q, Zeng J, Wang B, Gao W, Chen K, Yuan Z, Xu J, Tang D (2018) Effect of retention rate of fluorescent cellulose nanofibrils on paper properties and structure. Carbohydr Polym 186:73-81. https://doi.org/10.1016/j.carbpol.2018. 01.040

Enomae T, Han Y-H, Isogai A (2008) Z-directional distribution of fiber orientation of Japanese and western papers determined by confocal laser scanning microscopy. J Wood Sci 54:300-307. https://doi.org/10.1007/s10086-008-0950-z

Fischer W, Mayr M, Spirk S, Reishofer D, Jagiello L, Schmiedt R, Colson J, Zankel A, Bauer W (2017) Pulp finescharacterization, sheet formation, and comparison to microfibrillated cellulose. Polymers 9:366-378. https:// doi.org/10.3390/polym9080366

Giner Tovar R, Fischer WJ, Eckhart R, Bauer W (2015) White water recirculation method as a means to evaluate the influence of fines on the properties of handsheets. Bioresources 10:7242-7251. https://doi.org/10.15376/biores.10. 4.7242-7251

Hobisch MA, Müller D, Fischer W, Zankel A, Eckhart R, Bauer W, Zabler S, Spirk S (2019) Cobalt ferrite nanoparticles for three dimensional visualization of micro- and nanostructured cellulose in paper. ACS Appl Nano Mater. https://doi. org/10.1021/acsanm.9b00718

Latour G, Echard JP, Didier M, Schanne-Klein MC (2012) In situ 3D characterization of historical coatings and wood using multimodal nonlinear optical microscopy. Opt Express 20:24623-24635. https://doi.org/10.1364/OE.20. 024623

Lundquist K, Josefsson B, Nyquist G (1978) Analysis of lignin products by fluorescence spectroscopy. Holzforschung 32:27-32. https://doi.org/10.1515/hfsg.1978.32.1.27

Mark RE (1984) Structure and structural anisotropy. V. Dimensional characterization of shives, slivers, and fines. In: Mark RE (ed) Handbook of physical and mechanical testing of paper and paperboard, vol 2. Dekker, Philadelphia

Mayr M, Eckhart R, Bauer W (2017a) Improved microscopy method for morphological characterisation of pulp fines. Nord Pulp Pap Res J 32:244-252. https://doi.org/10.3183/ npprj-2017-32-02-p244-252

Mayr M, Eckhart R, Winter H, Bauer W (2017b) A novel approach to determining the contribution of the fiber and fines fraction to the water retention value (WRV) of chemical and mechanical pulps. Cellulose 24:3029-3036. https://doi.org/10.1007/s10570-017-1298-6

Moss PA, Retulainen E, Paulapuro H, Aaltonen P (1993) Taking a new look at pulp and paper: applications of confocal laser scanning microscopy to pulp and paper research. Pap Puu 75:74-79

Odabas N, Henniges U, Potthast A, Rosenau T (2016) Cellulosic fines: properties and effects. Prog Mater Sci 83:574-594. https://doi.org/10.1016/j.pmatsci.2016.07.006

Osong SH, Norgren S, Engstrand P (2015) Processing of woodbased microfibrillated cellulose and nanofibrillated cellulose, and applications relating to papermaking: a review. Cellulose 23:93-123. https://doi.org/10.1007/s10570-0150798-5

Retulainen E, Moss P, Nieminen K (1993) Effect of fines on the properties of fibre networks. Paper presented at the products of papermaking. 10th fundamental research symposium, Oxford 
TAPPI (1994) Fines fraction of paper stock by wet screening. Test method T $261 \mathrm{~cm}-94$

Westermark U, Capretti G (1988) Influence of ray cells on the bleachability and properties of CTMP and kraft pulps. Nord Pulp Pap Res J 03:095-099. https://doi.org/10.3183/ NPPRJ-1988-03-02-p095-099
Publisher's Note Springer Nature remains neutral with regard to jurisdictional claims in published maps and institutional affiliations. 